\title{
Differences in Motor Unit Recruitment Patterns and Low Frequency Oscillation of Discharge Rates between Unilateral and Bilateral Isometric Muscle Contractions.
}

\author{
Shancheng Bao \\ Texas A\&M University \\ Yiyu Wang \\ Texas A\&M University \\ David L Wright \\ Texas A\&M University \\ John J. Buchanan \\ Texas A\&M University \\ Yuming Lei ( $\nabla$ yx1907@tamu.edu ) \\ Texas A\&M University
}

\section{Research Article}

Keywords: bimanual contraction, motor unit, surface EMG decomposition, coefficients of variation, first common component, size principle

Posted Date: July 27th, 2021

DOl: https://doi.org/10.21203/rs.3.rs-753856/v1

License: (c) This work is licensed under a Creative Commons Attribution 4.0 International License.

Read Full License

Version of Record: A version of this preprint was published at Human Movement Science on June 1st, 2022. See the published version at https://doi.org/10.1016/j.humov.2022.102952. 
Differences in Motor Unit Recruitment Patterns and Low Frequency Oscillation of Discharge

Section and Senior Editor:

Abbreviated title: Motor unit behavior during unilateral and bilateral force generation

15 Number of figures: 6

16 Number of tables: 0

17 Number of pages: 30

Keywords: bimanual contraction, motor unit, surface EMG decomposition, coefficients of variation, first common component, size principle

Correspondence to:

Yuming Lei, PhD.

Department of Health and Kinesiology

Texas A\&M University

Phone: (979) 862-3392

E-mail:yx1907@tamu.edu 
Abstract

40 Introduction: Distinct cortical activities contribute to unilateral and bilateral motor control.

41 However, it remains largely unknown whether the behavior of motor neurons differs between

42 unilateral and bilateral isometric force generation. Here, we first investigated motor units (MUs)

43 recruitment patterns during unilateral and bilateral force generation. Considering that the force

44 control is primarily regulated by low-frequency synaptic inputs to motor neurons, we also

45 examined the relation between $\mathrm{MU}$ discharge rate and force output during unilateral and

46 bilateral muscle contractions. Methods: Using advanced electromyography (EMG) sensor arrays

47 and spike-triggered averaging techniques, we examined a large population of MUs in the right

48 first dorsal interosseous (FDI) muscle during unilateral and bilateral force tracking tasks. Using

49 the principal component analysis, we analyzed the first common component (FCC) of MU

50 discharge rate to describe the force fluctuations during unilateral and bilateral contractions.

51 Results: We found that MU discharge rate decreased during bilateral compared with unilateral

52 contractions. MU recruitment threshold increased, while the amplitude and duration of MU

53 action potential (MUAP) remained unchanged during bilateral compared with unilateral

54 contractions. We found that the coefficients of variation (CV) for the force and FCC signal

55 increased during bilateral compared with unilateral contractions. Notably, the FCC signal

56 captured a great amount of MU discharge variability, and its CV correlated with the CV of the

57 force signal. Conclusion: Our findings suggest that MU recruitment patterns are altered during

58 bilateral compared with unilateral isometric force generation, likely related to changes at the

59 low-frequency portion of the synaptic drive.

60 
63 control $^{1,2}$. For example, electrophysiological studies in non-human primates showed that cells in

64 cortical motor areas modulate their activities during unimanual and bimanual tasks ${ }^{3-6}$, and the

65 excitatory callosal inputs undergo suppression during bimanual tasks ${ }^{7}$. Primate lesion studies

66 demonstrated that unilateral lesion in the supplementary motor area (SMA) does not affect the

67 animal's general motor behavior, but produces long-lasting deficits in bimanual coordination ${ }^{8,9}$.

68 In humans, neurophysiological studies showed changes in corticospinal excitability and cortico-

69 cortical interactions during bilateral compared with unilateral force generation ${ }^{10-13}$.

70 Neuroimaging studies revealed that bilateral movements involve unique neural activation

71 patterns in the cortical motor network compared with unilateral movements ${ }^{14-17}$. Despite the

72 intensive investigation into the control of unilateral and bilateral movements at the cortical level,

73 the behavior of motor neurons in humans during unilateral and bilateral force control remains

74 poorly understood.

The combination of advanced electromyography (EMG) sensor arrays and signal

76 processing techniques has provided a means to examine the activity of a large population of

77 motor units (MUs) obtained from the innervated muscles non-invasively ${ }^{18-21}$. In this study, we

78 systematically assessed MU discharge rate, MU recruitment threshold, and the amplitude and

79 duration of MU action potential (MUAP) in the first dorsal interosseous (FDI) muscle during low-

80 force unilateral and bilateral contractions. Evidence has shown that MUs can be recruited in a

81 task-dependent manner $22-24$. For example, animal studies showed that separate groups of MUs

82 are selectively recruited for different movement conditions ${ }^{25-27}$. In humans, the recruitment of 
83 MUs can be altered by external stimulations ${ }^{28,29}$, and the brain can selectively stimulate the MUs

84 regardless of the size principle criterion ${ }^{22}$. Specifically, MUs in the FDI muscle are differentially

85 recruited for different hand movements ${ }^{20,23,24}$. Notably, common drive to MUs is reduced during

86 bilateral compared with unilateral FDI muscle contractions ${ }^{30}$. Moreover, a single limb during a

87 maximal bilateral contraction produces a lower force ${ }^{31,32}$, and a lesser activation of slow- and

88 fast-twitch MUs $\mathrm{M}^{33-35}$ compared with a maximal unilateral contraction. Based on the information,

89 we hypothesized that MU recruitment patterns differed between unilateral and bilateral

90 voluntary contractions.

91 The force production is accomplished by the transmission of synaptic inputs to motor

92 neurons. The pool of motor neurons receives both common and independent synaptic inputs

93 from supraspinal and spinal circuits ${ }^{36-39}$, and the force control is primarily regulated by low-

94 frequency common inputs to motor neurons ${ }^{40,41}$. In this study, we also investigated the low-

95 frequency oscillations of the MU discharge rate using the principal component analysis and

96 examined its relationship with the force signal during unilateral and bilateral contractions.

97 Evidence showed that the variability of the force output increases during bilateral compared with

98 unilateral force generation ${ }^{42,43}$, and the variability of low-frequency components of MU discharge

99 rate contribute to the variability of the force output ${ }^{41,44}$. Therefore, we also expected that low-

100 frequency synaptic inputs contribute to the force control during unilateral and bilateral

101 contractions.

102

103

Materials and Methods 
104 Subjects. Thirteen right-handed healthy volunteers ( $28.5 \pm 7.8$ years old, 7 females) participated

105 in the study. All subjects reported no history of neuromuscular disorders and were naïve to the

106 paradigm and the purpose of the study. All experimental protocols were approved by the

107 Institutional Review Board of Texas A\&M University. All subjects gave written informed consent

108 prior to participation, which was approved by the local ethics committee at Texas A\&M University

109 in accordance with the Declaration of Helsinki.

110 Electromyographic (EMG) recordings. EMG was recorded from the right FDI muscle through a

111 Trigno Galileo surface sensor array (Delsys, Inc., Natick, MA) secured to the skin over the belly of

112 each muscle. The array consists of four $(2 \times 2)$ cylindrical probes $(0.5 \mathrm{~mm}$ diameter $)$ and pairwise

113 differentiation of the four electrodes yields four channels of the EMG signals. The amplified

114 signals were converted to digital data with a sampling rate of $2222 \mathrm{~Hz}$ and stored in a computer

115 for offline analysis.

116 Experimental paradigm. Subjects were seated in a custom chair with both arms flexed at the

117 elbow by $90^{\circ}$ with the forearm pronated. The left and right index fingers were attached to two

118 load cells, which measured the forces exerted by the subjects. At the beginning of the

119 experiment, subjects performed two or three isometric maximal voluntary contractions (MVCs)

120 for 3-5 s with right and left index finger into abduction, separated by $60 \mathrm{~s}$ of rest. During maximal

121 contractions, subjects were verbally encouraged to perform maximally, and visual feedback was

122 provided. The maximal forces were used to set targets for subsequent submaximal contractions.

123 During testing, subjects were requested to perform a series of isometric voluntary contractions

124 with the right hand, while the left hand remained at rest or performed isometric voluntary 
125 contractions. Subjects were instructed to perform unilateral or bilateral contractions by following

126 a trapezoidal target force trajectory, which consisted of a linearly up-ramp from rest to the

127 prescribed \%MVC in $10 \mathrm{~s}$, a constant force of prescribed \%MVC for $10 \mathrm{~s}$, and a linearly down-

128 ramp to rest in $10 \mathrm{~s}$ (Fig. 1A). Two constant force levels ( $10 \%$ and $30 \%$ of MVC) were tested.

129 Custom software written by the MotionMonitor xGen (Innovative Sports Training, Inc. Chicago,

130 IL) was used to acquire force signals from the load cell and to display visual feedback

131 corresponding to the right and left FDI muscle force in real time (Innovative Sports Training, Inc.,

132 Chicago, USA). One or two cursors were used to indicate the current force generated by the right

133 or left FDI muscle, and the subjects were asked to maintain the target force as accurately as

134 possible (Fig. 1A). Familiarization trials were completed at the beginning of the experiment to

135 ensure that subjects were able to trace the trapezoid trajectory closely. Subjects performed two

136 trials of unilateral and bilateral contractions for each force level. The order of the contractions

137 was randomized for each subject. A 30 s rest period between the contractions was provided to

138 minimize fatigue. Note that the unilateral contractions were performed with the right hand, and

139 force output and EMG signals were recorded from the right FDI. The root mean squared error

140 (RMSE) was calculated to assess the force accuracy, which measured the deviation of the force

141 signal from the target force trajectory ${ }^{42}$. The coefficient of variation (CV) was calculated to assess

142 the variability of the force signal relative to the mean force output ${ }^{42}$.

143 EMG signal decomposition. The recorded EMG signals were decomposed into discriminable

144 motor units (MUs) using the decomposition algorithm introduced by De Luca ${ }^{18,21,45}$. For each

145 identified MU, the algorithm output consisted of MU discharge times and MUAP templates (Fig.

146 1B and C). MU waveforms (amplitude and shape) recorded from the surface electrodes were 
147 obtained by applying spike-triggered averaging (STA) on the raw surface EMG signals. Specifically,

148 the discharge times of each MU identified from the decomposition system were used as triggers

149 for the STA calculation. To ensure the reliability of the STA estimate ${ }^{20,46}$, two tests were

150 performed: (1) the estimation the CV of the MUAP amplitude, and (2) the maximum linear

151 correlation coefficient between the STA estimate and the decomposition-estimated templates.

152 The MUs with a correlation coefficient $>0.7$ and CV of MUAP amplitude $<0.3$ were retained, and

153 a total of $715 \mathrm{MUs}$ were included for further analysis. For each retained MU, the MUAP amplitude

154 of the STA template was used as an estimate for the amplitude of MU, measured as the voltage

155 difference from minimum and maximum peaks. The MUAP duration was estimated as the time

156 between the zero crossings before the first positive peak of MU and the zeros crossing after the

157 last positive peak. The recruitment threshold of the selected MU was measured from the

158 averaged isometric force data over the interval -50 to $150 \mathrm{~ms}$ relative to the instance of the first

159 discharge event. The peak discharge rate was calculated from the 4-s averaging window in which

160 the force was relatively constant at a steady state when the discharge rate was stable, while the

161 average discharge rate was calculated from the entire contraction duration. The inter-pulse

162 interval (IPI) was measured as the time interval between adjacent pulses.

163 Principal component analysis of the $\mathrm{MU}$ discharge rate. It has been demonstrated that the

164 effective neural drive to muscles is controlled by low-frequency synaptic inputs to motor

165 neurons ${ }^{47-49}$. To investigate the relationship between the $\mathrm{MU}$ discharge rate and force output

166 during unilateral and bilateral contractions, we analyzed the FCC of MU discharge rate using the

167 principal component analysis. It has been shown that the FCC signal captures a great amount of

168 variability in the smoothed $\mathrm{MU}$ discharge rate and explains a large part of fluctuation in the 
169 muscle force during contractions ${ }^{41,44}$. In this study, the smoothed and detrended MU discharge

170 rate were arranged in a matrix (time sequence $\times \mathrm{MU}$ ). The FCC of $\mathrm{MU}$ discharge rate was

171 computed by using the eigenvalue decomposition of the covariance matrix ${ }^{41}$, and the maximum

172 eigenvalue of the covariance matrix is used to quantify the FCC signal ${ }^{44}$. In addition, the CVs for

173 the FCC and force signal were computed as the ratio (\%) between standard deviation and the

174 mean values after removing the linear trends from the signals ${ }^{41,44}$.

175 Statistical analysis. The mean values of the threshold force, amplitude, duration, discharge rate,

176 and inter-pulse interval of the MUs were calculated during unilateral and bilateral contractions

177 for each subject. Regression analyses with a generalized linear model were conducted to examine

178 the relation between the amplitude and threshold force, as well as the relation between the

179 discharge rate and threshold force. In addition, an inverse power function was used to examine

180 the relation between the discharge rate and amplitude: discharge rate $=b \times(\text { amplitude })^{a}$, where

$181 \mathrm{~b}$ is the scale factor and $\mathrm{a}$ is the rate of decay. The fit slopes of the amplitude vs. threshold force,

182 the discharge rate vs. threshold force, and the discharge rate vs. P-P amplitude were calculated

183 at each contraction for each subject. Repeated repeated-measures ANOVAs were performed to

184 determine the effect of TASK (unilateral, bilateral) and FORCE ( $10 \%$ and $30 \%$ of MVC) on MU

185 threshold force, amplitude, duration, discharge rate, inter-pulse interval, and fit slope. The same

186 analysis was used to determine the effect of TASK and FORCE on the CV for the force and FCC

187 signal. Further, linear regression analysis was used to determine the relationship between the CV

188 for the FCC signal and the CV for the force signal. For all variables, normal distribution was tested

189 by the Shapiro-Wilk's test and homogeneity of variances by the Levene's test of equality and

190 Mauchly's test of sphericity. When normal distribution could not be assumed, data were log 
191 transformed. When sphericity could not be assumed, the Greenhouse-Geisser correction statistic

192 was used. Significance was set at $p<0.05$. Group data are presented as the mean \pm SD in the text.

\section{Results}

MU Discharge Rate

EMG signal decomposition identified a total of 715 motor units (MUs). The average

197 number of MUs obtained from the right FDI muscle per subject was $55.0 \pm 16.2$ (unilateral = 26.8

$198 \pm 8.2$; bilateral $=28.2 \pm 9.9, \mathrm{p}=0.6$ ). Figure $2 \mathrm{~A}$ illustrates the instantaneous $\mathrm{MU}$ discharge rate as

199 a function of time for the three representative MUs recorded during the unilateral (black) and

200 bilateral (grey) FDI muscle contractions at 10\% (Fig. 2A, left) and 30\% (Fig. 2A, right) of MVC. Note

201 that MU discharge rate in the right FDI muscle was suppressed during bilateral compared with

202 unilateral contractions.

Repeated-measures ANOVA showed an effect of TASK $\left(F_{(1,12)}=15.9, p=0.002\right)$ and FORCE

$204\left(F_{(1,12)}=198.2, p<0.001\right)$, but not in their interaction $\left(F_{(1,12)}=0.3, p=0.6\right)$ on the peak MU

205 discharge rate. Post hoc analysis revealed that the peak discharge rate of the identified MUs

206 decreased significantly during bilateral $(10 \%$ of $M V C=12.9 \pm 2.3$ pulses per second (pps); $30 \%$ of

$207 \mathrm{MVC}=18.8 \pm 3.0 \mathrm{pps})$ compared with unilateral $(10 \%$ of $\mathrm{MVC}=13.9 \pm 2.2 \mathrm{pps} ; 30 \%$ of $\mathrm{MVC}=20.0$

$208 \pm 2.9 \mathrm{pps})$ contractions at $10 \%(p=0.002)$ and $30 \%(p=0.004)$ of MVC (Fig. $2 C)$. Another repeated-

209 measures ANOVA revealed an effect of TASK $\left(F_{(1,12)}=11.1, p=0.006\right)$ and FORCE $\left(F_{(1,12)}=129.3\right.$,

$210 p<0.001)$, but not in their interaction $\left(F_{(1,12)}=0.007, p=0.9\right)$ on the average MU discharge rate.

211 The average discharge rate showed a decline during bilateral ( $10 \%$ of MVC $=4.7 \pm 1.1 \mathrm{pps} ; 30 \%$

212 of $\mathrm{MVC}=6.5 \pm 1.0 \mathrm{pps})$ compared with unilateral $(10 \%$ of $\mathrm{MVC}=5.2 \pm 1.0 \mathrm{pps} ; 30 \%$ of $\mathrm{MVC}=7.0$ 
$213 \pm 1.2 \mathrm{pps})$ contractions at $10 \%(p=0.002)$ and $30 \%(p=0.01)$ of MVC (Fig. 2D). Repeated-

214 measures ANOVA also showed an effect of $\operatorname{TASK}\left(\mathrm{F}_{(1,12)}=8.5, \mathrm{p}=0.01\right)$ and $\mathrm{FORCE}\left(\mathrm{F}_{(1,12)}=49.5\right.$,

$215 p<0.001)$, but not in their interaction $\left(F_{(1,12)}=2.1, p=0.2\right)$ on the inter-pulse interval (IPI). The

216 IPI was significantly higher during bilateral $(10 \%$ of $\mathrm{MVC}=175.6 \pm 44.1 \mathrm{~ms} ; 30 \%$ of $\mathrm{MVC}=118.6$

$217 \pm 17.3 \mathrm{~ms})$ compared with unilateral $(10 \%$ of $\mathrm{MVC}=150.8 \pm 30.0 \mathrm{~ms} ; 30 \%$ of $\mathrm{MVC}=107.6 \pm 19.6$

$218 \mathrm{~ms})$ contractions at $10 \%(p=0.01)$ and $30 \%(p=0.02)$ of MVC (Fig. 2E).

219 MU Recruitment Threshold and MUAP Amplitude and Duration

220 Repeated-measures ANOVA revealed an effect of TASK $\left(F_{(1,12)}=11.1, p=0.006\right)$ and

221 FORCE $\left(F_{(1,12)}=99.0, p<0.001\right)$, but not in their interaction $\left(F_{(1,12)}=0.5, p=0.5\right)$ on the $M U$

222 recruitment threshold force. Post hoc analysis revealed that the MU recruitment threshold force

223 was higher during bilateral compared with unilateral contractions at $10 \%$ (bilateral $=1.5 \pm 0.9 \%$

224 MVC; unilateral $=1.1 \pm 0.5 \%$ MVC; $p=0.02)$ and $30 \%$ (bilateral $=5.5 \pm 1.7 \% \mathrm{MVC}$; unilateral $=$

$2254.7 \pm 1.4 \%$ MVC; $p=0.03$; Fig. 3A) of MVC. Repeated-measures ANOVAs showed no effect of

226 TASK on the duration $\left(F_{(1,12)}=0.04, p=0.9\right)$ and amplitude $\left(F_{(1,12)}=0.5, p=0.5\right)$ of the MUAP.

227 These results indicate that distinct MU recruitment patterns may be employed during unilateral

228 and bilateral muscle contractions. No changes were observe in the duration of the MUAP during

229 unilateral $(10 \%$ of $\mathrm{MVC}=10.43 \pm 0.96 \mathrm{~ms} ; 30 \%$ of $\mathrm{MVC}=9.56 \pm 0.89 \mathrm{~ms})$ and bilateral $(10 \%$ of

$230 \quad M V C=10.62 \pm 1.20 \mathrm{mV} ; 30 \%$ of $M V C=9.61 \pm 1.02 \mathrm{mV})$ contractions at $10 \%(p=0.6)$ and $30 \%(p$

$231=0.8$ ) of MVC (Fig. 3B). The amplitude of the identified MUs was not significantly different

232 between unilateral $(10 \%$ of $\mathrm{MVC}=0.104 \pm 0.06 \mathrm{mV} ; 30 \%$ of $\mathrm{MVC}=0.226 \pm 0.12 \mathrm{mV})$ and bilateral

$233(10 \%$ of $\mathrm{MVC}=0.101 \pm 0.04 \mathrm{mV} ; 30 \%$ of $\mathrm{MVC}=0.229 \pm 0.17 \mathrm{mV})$ contractions at $10 \%(p=0.4)$

234 and $30 \%(p=0.4)$ of MVC (Fig. 3C). 
Size Principle

Figure 4A illustrates $\mathrm{MU}$ recruitment threshold force as a function of $\mathrm{MU}$ amplitude

237 during unilateral and bilateral contractions at 10\% (Fig. 4A, left) and 30\% (Fig. 4A, right) of MVC

238 in a representative subject. The fit lines for each contraction are plotted. Note that there was a

239 linear relationship between the threshold force and MU amplitude during unilateral (black) and

240 bilateral (grey) contractions. Figure 4B shows the discharge rate in relation to MU amplitude.

241 There was an inverse power relationship between the discharge rate and MU amplitude. Figure

242 4C illustrates the threshold force as a function of the discharge rate, with regression lines

243 superimposed on the motor units. An inverse linear relation was found between the discharge

244 rate and threshold force. No differences were observed in the fit slop for threshold force vs. MU

245 amplitude (10\% of MVC: unilateral $=0.044 \pm 0.032$, bilateral $=0.040 \pm 0.033, p=0.4 ; 30 \%$ of MVC:

246 unilateral $=0.024 \pm 0.008$, bilateral $=0.026 \pm 0.017, \mathrm{p}=0.3), \mathrm{MU}$ amplitude vs. discharge rate

$247(10 \%$ of MVC: unilateral $=-0.81 \pm 0.14$, bilateral $=-0.84 \pm 0.13, p=0.4 ; 30 \%$ of $\mathrm{MVC}:$ unilateral $=$

$248-0.45 \pm 0.13$, bilateral $=-0.48 \pm 0.12, p=0.2)$, and threshold force vs. discharge rate $(10 \%$ of MVC:

249 unilateral $=-2.26 \pm 1.17$, bilateral $=-2.09 \pm 1.33, \mathrm{p}=0.7 ; 30 \%$ of MVC: unilateral $=-1.32 \pm 0.54$,

250 bilateral $=-1.35 \pm 0.61, p=0.5)$ between unilateral and bilateral contractions. Together, these

251 data demonstrate that there was orderly recruitment of MUs based on MU size across a

252 population of concurrently active MUs, regardless of unilateral or bilateral contractions.

254 Low-frequency oscillation of the MU discharge rate

255 The low-frequency oscillations of MU discharge rate were examined by computing the 256 FCC of MU spike trains ${ }^{41,44}$. Figure $5 A$ and B illustrate the FCC signal of MU discharge rate (black) 257 and the force signal (grey) during unilateral and bilateral contractions in a representative subject. 
258 Note that the fluctuation of the force signal is greater during bilateral compared with unilateral 259 contractions and the force and FCC signals have similar low-frequency oscillations. The FCC signal 260 explained a great amount of the total variability of MU discharge rate during unilateral (10\% of $261 \mathrm{MVC}=43.2 \pm 12.1 \% ; 30 \%$ of $\mathrm{MVC}=39.5 \pm 11.3 \%)$ and bilateral $(10 \%$ of MVC $=43.4 \pm 9.3 \% ; 30 \%$

262 of MVC $=44.1 \pm 11.2 \%)$ contractions. The FCC signal accounted for the most variability of MU 263 discharge rate was similar between unilateral and bilateral contractions at $10 \%(p=0.5)$ and $30 \%$ $264(p=0.2)$ of MVC. Repeated-measures ANOVA showed an effect of $\operatorname{TASK}\left(F_{(1,12)}=10.2, p=0.008\right)$ 265 and FORCE $\left(F_{(1,12)}=0.4, p=0.5\right)$, but not in their interaction $\left(F_{(1,12)}=0.2, p=0.6\right)$ on the CV of the 266 FCC signal. Post hoc analysis showed that FCC signal quantified with the CV was greater in the 267 bilateral contractions $(10 \%$ of $M V C=4.59 \pm 1.38 \% ; 30 \%$ of $\mathrm{MVC}=4.92 \pm 1.69 \%)$ than in the 268 unilateral contractions $(10 \%$ of $M V C=4.06 \pm 1.28 \% ; 30 \%$ of $M V C=4.17 \pm 1.08 \%)$ at $10 \%(p=$ 2690.03 ) and 30\% ( $p=0.02$ ) of MVC (Fig. 5C). Similarly, repeated-measures ANOVAs revealed an 270 effect of TASK $\left(F_{(1,12)}=14.8, p=0.02\right)$ on the CV of the force signal. Post hoc analysis showed that 271 the CV of the force signal was greater during bilateral $(10 \%$ of $\mathrm{MVC}=3.52 \pm 1.14 \% ; 30 \%$ of $\mathrm{MVC}$ $272=2.85 \pm 1.33 \%)$ compared with unilateral $(10 \%$ of $\mathrm{MVC}=2.72 \pm 0.74 \% ; 30 \%$ of $\mathrm{MVC}=2.28 \pm$ $2730.72 \%)$ contractions at $10 \%(p=0.008)$ and $30 \%(p=0.04)$ of MVC (Fig. 5D). Notably, we found a 274 correlation between the CVs for the force and FCC signal ( $r=0.43, p=0.002 ;$ Fig. 6). This result 275 indicates that individuals with more force fluctuation were the ones with larger variability in low276 frequency oscillations of MU discharge rate. 
280 and low-frequency common presynaptic inputs to motor neurons during bilateral and unilateral

281 force control. Specifically, we found that MU discharge rate decreased, while MU recruitment

282 threshold increased, during bilateral compared with unilateral contractions. Motor neurons

283 receive low-frequency common presynaptic inputs during the force control. Therefore, we also

284 examined low-frequency components of the neural signal (FCC), reflecting the effective

285 primary/principal neural drive to the muscle. We found that the variability of the force and FCC

286 signal increased during bilateral compared with unilateral contraction. Notably, the FCC signal

287 explained most of MU discharge rate variability, and its CV was significantly correlated with the

288 CV of the force signal. Taken together, we propose that changes at the low-frequency portion of 289 the synaptic drive may contribute to alternations in MU recruitment patterns during bilateral 290 muscle contractions.

291 Motor Unit Recruitment Patterns during Unilateral and Bilateral Muscle Contractions

292 Extensive evidence in animals and humans showed that distinct cortical activities

293 contribute to unilateral and bilateral force control ${ }^{3-7,10-13,16,17}$. For example, bilateral motor tasks

294 result in higher neural activation in the primary sensorimotor cortex and supplementary motor

295 area compared with unilateral motor tasks ${ }^{16}$. M1 neurons are selectively fired during unilateral

296 and bilateral movements ${ }^{50,51}$. In this study, we reported that the nervous system might also

297 employ distinct MU recruitment strategies during bilateral compared with unilateral

298 contractions. We suggest that two mechanisms potentially contribute to altered behavior of

299 motor neurons during unilateral and bilateral contractions: (1) changes in the recruitment order 
300 of MUs, and (2) changes in the population of MUs recruited during unilateral and bilateral 301 contractions.

302 It is commonly assumed that the "size principle" rule, orderly recruitment of MUs based 303 on MU size, governs the sections of MUs for voluntary muscle contractions ${ }^{52-56}$. However, some 304 evidence has shown that the orderly recruitment of MUs can be altered based on the context of 305 the movement tasks ${ }^{29,57}$. For example, human studies showed that the nervous system can learn 306 to control individual MUs regardless of their size 22,58 , indicating that the recruitment order of 307 MUs can be flexible. MUs can change their recruitment order when voluntary contractions are 308 performed in different directions ${ }^{23,59}$, positions $s^{60,61}$, and velocity levels ${ }^{57}$. Further, animal studies 309 showed that rapid locomotor movements can result in non-orderly recruitment of MUs ${ }^{62-64}$. The

310 existence of orderly recruitment enables fatigue-resistant muscle contractions, but poses a 311 mechanical paradox. In contrast, a random recruitment order of MUs would be mechanically 312 effective because it can deploy the specific MUs to satisfy the mechanical demands of the motor 313 tasks in an efficient way. Our results showed orderly recruitment of MUs, with the discharge rate 314 of earlier-recruited MUs being greater than those later-recruited MUs, during both unilateral and 315 bilateral contractions, suggesting that the "size principle" rule can provide a general description 316 of MU recruitment patterns, regardless of unilateral or bilateral contractions for this task.

317 An alternative mechanism is that two different populations of MUs in the FDI muscle may 318 be recruited during unilateral and bilateral contractions. Evidence has shown that the nervous 319 system can select distinct MU populations for different movements. For example, in walking cats, 320 separate groups of MUs are selectively recruited for different movement conditions ${ }^{25-27}$. 
321 Electrophysiological studies in humans revealed that different populations of lower limb MUs are

322 recruited in a task-dependent manner during locomotion ${ }^{65}$. In the FDI muscle, MUs can also be

323 differentially recruited for different hand movements $20,23,24$. Thus, it is possible that the

324 population of MUs recruited during bilateral contractions is modified. Indeed, our findings

325 showed that MU discharge rate decreased during bilateral compared with unilateral

326 contractions. This aligns with the previous findings showing that the activation of slow- and fast-

327 twitch MUs decreases during bilateral compared with unilateral maximal contractions ${ }^{33-35}$. To

328 maintain the designated force output during bilateral contractions, the reduced discharge rate

329 can be offset by the recruitment of new MUs. This agrees with evidence showing that changes in

330 MU populations can compensate for reduced MU discharge rate to maintain force ${ }^{66}$. Further, we

331 showed that the MU recruitment threshold increased during bilateral compared with unilateral

332 contractions. Since the recruitment threshold varies for different motor units in which larger MUs

333 are typically recruited at higher threshold force while smaller MUs are recruited at lower

334 threshold force, this result may also suggest that the recruited MUs during bilateral contractions

335 are not the same MUs recruited during unilateral contractions. Thus, we suggest that separate

336 populations of MUs in the FDI may be selectively activated to produce a given force during

337 unilateral and bilateral contractions, and distinct MUs may contribute differently to the force

338 output during these two contraction conditions.

339 Low-frequency oscillation of MU discharge rate

340 The motoneuron pool receives common and independent presynaptic inputs from

341 supraspinal and spinal circuitries ${ }^{36-39}$, and low-frequency common synaptic inputs determine the

342 force control ${ }^{40,67}$. The next question is to address if common inputs to motor neurons, during 
343 bilateral contractions, were different from those present during unilateral contractions. Here,

344 the principal component analysis of the smoothed MU spike trains (the FCC signal) was used to

345 provide insights into the low-frequency oscillation of the neural drive to the FDI muscle. Indeed,

346 we found that low-frequency oscillations of MU discharge rate were well represented by the FCC

347 signal extracted by principal component analysis during ( $10 \%$ of $\mathrm{MVC}=43.2 \pm 12.1 \%$; $30 \%$ of $\mathrm{MVC}$

$348=39.5 \pm 11.3 \%)$ and bilateral $(10 \%$ of $\mathrm{MVC}=43.4 \pm 9.3 \% ; 30 \%$ of $\mathrm{MVC}=44.1 \pm 11.2 \%)$

349 contractions. The FCC signal likely reflects the effective drive to the muscle because it captures

350 low-frequency oscillations of the force signal ${ }^{40,41,44}$. Here, we reported an increased variability

351 (CV) of the force signal during bilateral compared with unilateral contractions, demonstrating a

352 poorer force control during the bilateral tasks. Our findings are consistent with previous

353 behavioral studies showing that the bimanual force output was more variable with respect to

354 unilateral conditions ${ }^{42,43}$. In addition, our results showed that the variability of the FCC signal was

355 greater in the bilateral contractions than in the unilateral contractions, indicating an increased

356 variability in the smoothed discharge rate during bilateral contractions. It was previously shown

357 that the low-frequency oscillations of MU discharge rate regulate force steadiness and the FCC

358 signal captures most of the force fluctuation ${ }^{41,44}$. Thus, the increased variability in the FCC signal

359 may be related to the reduced ability of the nervous system to produce a stable neural drive to

360 the muscle during bilateral contractions. This is in agreement with previous studies showing that

361 the effective neural drive to muscles was reduced during a maximal bilateral contraction ${ }^{33-35,68}$.

362 This is also supported by the high correlation found between the CV for the FCC signal and the

363 CV for the force signal, in which we found that participants with more variability of the FCC signal

364 also showed larger force fluctuation. This result further confirms that the low-frequency 
365 oscillatory component of the MU discharge rate has a great influence on force fluctuation.

366 Together, our results indicate that the differences in force variability during unilateral and

367 bilateral contractions could be attributed to changes at the low-frequency components of

368 synaptic inputs to motor neurons.

369 Conclusion

370 In conclusion, we analyzed the behavior of motor neurons and low-frequency common

371 synaptic inputs to motor neurons during unilateral and bilateral contractions. Understanding the

372 bilateral control properties regulating motor neuron behavior and common synaptic inputs is

373 important for understanding the mechanisms underlying motor abnormality. Thus, our results

374 are particularly relevant for individuals with neurological disorders, such as stroke ${ }^{69-71}$ and spinal

375 cord injury ${ }^{72,73}$, since bilateral training is a common motor rehabilitation strategy for those

376 patients. Therefore, our findings can provide the background information for developing

377 rehabilitation strategies for patients with stroke and spinal cord injury. 


\section{References}

1. Carson RG. Neural pathways mediating bilateral interactions between the upper limbs. Brain Res Brain Res Rev. 2005;49(3):641-662.

2. Donchin O, de Oliveira SC, and Vaadia E. Who tells one hand what the other is doing: the neurophysiology of bimanual movements. Neuron. 1999;23(1):15-18.

3. Cardoso de Oliveira S, Gribova A, Donchin O, Bergman H, and Vaadia E. Neural interactions between motor cortical hemispheres during bimanual and unimanual arm movements. Eur J Neurosci. 2001;14(1):1881-1896.

4. Donchin O, Gribova A, Steinberg O, Mitz AR, Bergman H, and Vaadia E. Single-unit activity related to bimanual arm movements in the primary and supplementary motor cortices. J Neurophysiol. 2002;88(6):3498-3517.

5. Soteropoulos DS, Edgley SA, and Baker SN. Lack of evidence for direct corticospinal contributions to control of the ipsilateral forelimb in monkey. $J$ Neurosci. 2011;31(31):11208-11219.

6. Tanji J, Okano K, and Sato KC. Neuronal activity in cortical motor areas related to ipsilateral, contralateral, and bilateral digit movements of the monkey. J Neurophysiol. 1988;60(1):325-343.

7. Rokni U, Steinberg O, Vaadia E, and Sompolinsky H. Cortical representation of bimanual movements. J Neurosci. 2003;23(37):11577-11586.

8. Brinkman C. Lesions in supplementary motor area interfere with a monkey's performance of a bimanual coordination task. Neurosci Lett. 1981;27(3):267-270.

9. Brinkman C. Supplementary motor area of the monkey's cerebral cortex: short- and longterm deficits after unilateral ablation and the effects of subsequent callosal section. $J$ Neurosci. 1984;4(4):918-929.

10. Long J, Tazoe T, Soteropoulos DS, and Perez MA. Interhemispheric connectivity during bimanual isometric force generation. J Neurophysiol. 2016;115(3):1196-1207.

11. Sohn YH, Kaelin-Lang A, and Hallett M. The effect of transcranial magnetic stimulation on movement selection. J Neurol Neurosurg Psychiatry. 2003;74(7):985-987.

12. Yamaguchi T, Moriya K, Tanabe S, Kondo K, Otaka Y, and Tanaka S. Transcranial directcurrent stimulation combined with attention increases cortical excitability and improves motor learning in healthy volunteers. J Neuroeng Rehabil. 2020;17(1):23.

13. Yedimenko JA, and Perez MA. The effect of bilateral isometric forces in different directions on motor cortical function in humans. J Neurophysiol. 2010;104(6):2922-2931.

14. Grefkes C, Eickhoff SB, Nowak DA, Dafotakis M, and Fink GR. Dynamic intra- and interhemispheric interactions during unilateral and bilateral hand movements assessed with fMRI and DCM. Neuroimage. 2008;41(4):1382-1394.

15. Nair DG, Purcott KL, Fuchs A, Steinberg F, and Kelso JA. Cortical and cerebellar activity of the human brain during imagined and executed unimanual and bimanual action sequences: a functional MRI study. Brain Res Cogn Brain Res. 2003;15(3):250-260.

16. Noble JW, Eng JJ, and Boyd LA. Bilateral motor tasks involve more brain regions and higher neural activation than unilateral tasks: an fMRI study. Exp Brain Res. 2014;232(9):27852795. 
17. Theorin A, and Johansson RS. Zones of bimanual and unimanual preference within human primary sensorimotor cortex during object manipulation. Neuroimage. 2007;36 Suppl 2: T2-T15.

18. De Luca CJ, Adam A, Wotiz R, Gilmore LD, and Nawab SH. Decomposition of surface EMG signals. J Neurophysiol. 2006;96(3):1646-1657.

19. Hu X, Suresh AK, Li X, Rymer WZ, and Suresh NL. Impaired motor unit control in paretic muscle post stroke assessed using surface electromyography: a preliminary report. In: 2012 Annual International Conference of the IEEE Engineering in Medicine and Biology SocietyIEEE. 2012;p.4116-4119.

20. Lei Y, Suresh NL, Rymer WZ, and Hu X. Organization of the motor-unit pool for different directions of isometric contraction of the first dorsal interosseous muscle. Muscle Nerve. 2018;57(1):E85-E93.

21. Nawab SH, Chang SS, and De Luca CJ. High-yield decomposition of surface EMG signals. Clin Neurophysiol. 2010;121(10):1602-1615.

22. Basmajian JV. Control and training of individual motor units. Science. 1963;141(3579):440-441.

23. Desnedt $\mathrm{H}$, and Gidaux E. Spinal motoneuron recruitment in man: Rank deordering with direction but not with speed of voluntary movement. Science. 1981;214(4523):933-936.

24. Enoka RM, Robinson GA, and Kossev AR. Task and fatigue effects on low-threshold motor units in human hand muscle. J Neurophysiol. 1989;62(6):1344-1359.

25. Hoffer JA, Loeb GE, and Pratt CA. Single unit conduction velocities from averaged nerve cuff electrode records in freely moving cats. J Neurosci Methods. 1981;4(3):211-225.

26. Loeb GE, and Hoffer JA. Activity of spindle afferents from cat anterior thigh muscles. II. Effects of fusimotor blockade. J Neurophysiol. 1985;54(3):565-577.

27. Loeb GE, Hoffer JA, and Marks WB. Activity of spindle afferents from cat anterior thigh muscles. III. Effects of external stimuli. J Neurophysiol. 1985;54(3):578-591.

28. Stephens JA, Garnett R. Buller NP. Reversal of recruitment order of single motor units produced by cutaneous stimulation during voluntary muscle contraction in man. Nature. 1978;272(5651):362-364.

29. Hodson-Tole EF, and Wakeling JM. Motor unit recruitment for dynamic tasks: current understanding and future directions. J Comp Physiol B. 2009;179(1):57-66.

30. Marsden JF, Farmer SF, Halliday DM, Rosenberg JR, Brown P. The unilateral and bilateral control of motor unit pairs in the first dorsal interosseous and paraspinal muscles in man. J Physiol. 1999;521(Pt 2):553-564.

31. Jakobi JM, and Chilibeck PD. Bilateral and unilateral contractions: possible differences in maximal voluntary force. Can J Appl Physiol. 2001;26(1):12-33.

32. Skarabot J, Cronin N, Strojnik V, and Avela J. Bilateral deficit in maximal force production. Eur J Appl Physiol. 2016;116(11-12):2057-2084.

33. Oda S, and Moritani T. Maximal isometric force and neural activity during bilateral and unilateral elbow flexion in humans. Eur J Appl Physiol Occup Physiol. 1994;69(3):240-243.

34. Secher NH, Rorsgaard S, and Secher $\mathrm{O}$. Contralateral influence on recruitment of curarized muscle fibres during maximal voluntary extension of the legs. Acta Physiol Scand. 1978;103(4):456-462. 
35. Vandervoort AA, Sale DG, and Moroz J. Comparison of motor unit activation during unilateral and bilateral leg extension. J Appl Physiol Respir Environ Exerc Physiol. 1984;56(1):46-51.

36. De Luca CJ, and Erim Z. Common drive of motor units in regulation of muscle force. Trends Neurosci. 1994;17(7):299-305.

37. Farina D, Negro F, and Jiang N. Identification of common synaptic inputs to motor neurons from the rectified electromyogram. J Physiol. 2013;591(10):2403-2418.

38. Farmer SF, Bremner FD, Halliday DM, Rosenberg JR, and Stephens JA. The frequency content of common synaptic inputs to motoneurones studied during voluntary isometric contraction in man. J Physiol. 1993;470:127-155.

39. Lemon RN, Mantel GW, and Muir RB. Corticospinal facilitation of hand muscles during voluntary movement in the conscious monkey. J Physiol. 1986;381:497-527.

40. Farina D, and Negro F. Common synaptic input to motor neurons, motor unit synchronization, and force control. Exerc Sport Sci Rev. 2015;43(1):23-33.

41. Negro F, Holobar A, and Farina D. Fluctuations in isometric muscle force can be described by one linear projection of low-frequency components of motor unit discharge rates. $J$ Physiol. 2009;587(Pt 24):5925-5938.

42. Patel P, Zablocki V, and Lodha N. Bimanual force control differs between increment and decrement. Neurosci Lett. 2019;701:218-225.

43. Smits-Engelsman BC, Van Galen GP, and Duysens J. Force levels in uni-and bimanual isometric tasks affect variability measures differently throughout lifespan. Motor Control. 2004;8(4):437-449.

44. Farina D, Negro F, Gizzi L, and Falla D. Low-frequency oscillations of the neural drive to the muscle are increased with experimental muscle pain. I Neurophysiol. 2012;107(3):958-965.

45. De Luca CJ, and Hostage EC. Relationship between firing rate and recruitment threshold of motoneurons in voluntary isometric contractions. J Neurophysiol. 2010;104(2):10341046.

46. Hu X, Rymer WZ, and Suresh NL. Assessment of validity of a high-yield surface electromyogram decomposition. J Neuroeng Rehabil. 2013;10:99.

47. De Luca CJ, and Erim Z. Common drive in motor units of a synergistic muscle pair. $J$ Neurophysiol. 2002;87(4):2200-2204.

48. De Luca CJ, Gonzalez-Cueto JA, Bonato P, and Adam A. Motor unit recruitment and proprioceptive feedback decrease the common drive. J Neurophysiol. 2009;101(3):16201628.

49. Farina D, Jiang N, Rehbaum H, Holobar A, Graimann B, Dietl H, and Aszmann OC. The extraction of neural information from the surface EMG for the control of upper-limb prostheses: emerging avenues and challenges. IEEE Trans Neural Syst Rehabil Eng. 2014;22(4):797-809.

50. Donchin O, Gribova A, Steinberg O, Bergman H, Vaadia E. Primary motor cortex in involved in bimanual coordination. Nature. 1998;395(6699):274-278. 
51. Steinberg O, Donchin O, Gribova A, Cardosa de Oliveira S, Bergman H, Vaadia E. Neuronal populations in primary motor cortex encode bimanual arm movements. Eur J Neurosci. 2002;15(8):1371-1380.

52. Henneman E. Relation between size of neurons and their susceptibility to discharge. Science. 1957;126(3287):1345-1347.

53. Henneman E, Somjen G, and Carpenter DO. Functional Significance of Cell Size in Spinal Motoneurons. J Neurophysiol. 1965;28:560-580.

54. Milner-Brown HS, Stein RB, and Yemm R. Changes in firing rate of human motor units during linearly changing voluntary contractions. J Physiol. 1973;230(2):371-390.

55. Milner-Brown HS, Stein RB, and Yemm R. The contractile properties of human motor units during voluntary isometric contractions. J Physiol. 1973;228(2):285-306.

56. Milner-Brown HS, Stein RB, and Yemm R. The orderly recruitment of human motor units during voluntary isometric contractions. J Physiol. 1973;230(2):359-370.

57. Wakeling JM, Uehli K, Rozitis Al. Muscle fibre recruitment can respond to the mechanics of the muscle contraction. J R Soc Interface. 2006;3(9):533-554.

58. Wagman IH, Pierce DS, Burger RE. Proprioceptive influence, in volitional control of individual motor units. Nature. 1965;207(5000):957-958.

59. Thomas CK, Ross BH, and Stein RB. Motor-unit recruitment in human first dorsal interosseous muscle for static contractions in three different directions. J Neurophysiol. 1986;55(5):1017-1029.

60. Person RS. Rhythmic activity of a group of human motoneurones during voluntary contraction of a muscle. Electroencephalogr Clin Neurophysiol. 1974;36(6):585-595.

61. ter Haar Romeny B, Van Der Gon JD, and Gielen C. Changes in recruitment order of motor units in the human biceps muscle. Exp Neurol. 1982;78(2):360-368.

62. Hodson-Tole EF, Wakeling JM. Motor unit recruitment patterns 1: responses to changes in locomotor velocity and incline. J Exp Biol. 2008;211(Pt 12):1882-1892.

63. Hodson-Tole EF, Wakeling JM. Motor unit recruitment patterns 2: the inXuence on myoelectric intensity and muscle fascicle strain rate. J Exp Biol. 2008;211(Pt 12):18931902.

64. Lee SS, de Boef Miara M, Arnold AS, Biewener AA, and Wakeling JM. Recruitment of faster motor units is associated with greater rates of fascicle strain and rapid changes in muscle force during locomotion. J Exp Biol. 2013;216(Pt 2):198-207.

65. Wakeling JM. Motor units are recruited in a task-dependent fashion during locomotion. $J$ Exp Biol. 2004;207(Pt 22):3883-3890.

66. Tucker K, Butler J, Graven-Nielsen T, Riek S, and Hodges P. Motor unit recruitment strategies are altered during deep-tissue pain. J Neurosci. 2009;29(35):10820-10826.

67. Farina D, Negro F, Muceli S, and Enoka RM. Principles of motor unit physiology evolve with advances in technology. Physiology. 2016;31(2):83-94.

68. van Dieën JH, Cholewicki J, and Radebold A. Trunk muscle recruitment patterns in patients with low back pain enhance the stability of the lumbar spine. Spine (Phila Pa 1976). 2003;28(8): 834-841. 
69. Kwakkel G, Kollen BJ, van der Grond J, and Prevo AJ. Probability of regaining dexterity in the flaccid upper limb: impact of severity of paresis and time since onset in acute stroke. Stroke. 2003;34(9):2181-2186.

70. Nakayama H, Jorgensen HS, Raaschou HO, and Olsen TS. Compensation in recovery of upper extremity function after stroke: the Copenhagen Stroke Study. Arch Phys Med Rehabil. 1994;75(8):852-857.

71. Nakayama H, Jorgensen HS, Raaschou HO, and Olsen TS. Recovery of upper extremity function in stroke patients: the Copenhagen Stroke Study. Arch Phys Med Rehabil. 1994;75(4): 394-398.

72. Lei $Y$, and Perez MA. Phase-dependent deficits during reach-to-grasp after human spinal cord injury. J Neurophysiol. 2018;119(1):251-261.

73. Oxland TR, Bhatnagar T, Choo AM, Dvorak MF, Tetzlaff W, and Cripton PA. Biomechanical aspects of spinal cord injury. In: Neural tissue biomechanicsSpringer, 2010, p.159-180.

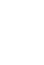

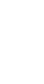




\section{Figure legends}

582 Figure 1. A, diagram showing the visual display presented to all subjects during testing of 583 unilateral and bilateral contractions. Subjects were instructed to remain at rest with the left hand 584 while the right hand was at rest or performed 10 or $30 \%$ of maximal voluntary contraction (MVC) 585 during index finger abduction. The trapezoids represent the trajectories that subjects needed to 586 keep the cursor in. The trapezoid width (geometry height of the trapezoid) represents the 587 magnitude of force required to accomplish each task, normalized to the maximal index finger 588 abduction determined in each subject. B, Motor unit recruitment threshold and discharge rate 589 during an isometric contraction of the first dorsal interosseous (FDI) muscle (plateau 10\% of MVC)

590 in a representative subject. The arrows highlight the recruitment of identified motor units. The 591 black line represents the net force output. C, The smoothed discharge rate of six representative 592 motor units.

593 Figure 2. A, The instantaneous motor unit discharge rate as a function of time for the three 594 representative motor units during unilateral (black) and bilateral (grey) contractions. B, Group 595 data $(n=13)$ showing peak discharge rate during unilateral and bilateral contractions. Data from 596 individual subjects are shown. C, Group data $(n=13)$ showing average discharge rate during 597 unilateral and bilateral contractions. Data from individual subjects are shown. D, Group data $598(n=13)$ showing inter pulse interval during unilateral and bilateral contractions. Data from 599 individual subjects are shown. Error bars indicate SEs. ${ }^{*} \mathrm{P}<0.05$, comparison between unilateral 600 and bilateral contractions. 
601 Figure 3. A, Motor unit recorded from the FDI muscle. The amplitude and duration of motor unit

602 action potential (MUAP) were measured as shown by the arrows. B, Group data $(n=13)$ showing

603 MUAP amplitude during unilateral and bilateral contractions. C, Group data $(\mathrm{n}=13)$ showing

604 MUAP duration during unilateral and bilateral contractions. D, Group data $(n=13)$ showing MU

605 recruitment threshold during unilateral and bilateral contractions. Error bars indicate SEs.

$606 * \mathrm{P}<0.05$, comparison between unilateral and bilateral contractions.

607 Figure 4. A, MUAP amplitude as a function of the threshold force at each contraction in a 608 representative subject. Linear regression lines are drawn through the data at each contraction.

609 B, MU discharge rate as a function of MUAP amplitude at each contraction in a representative 610 subject. An individual power function is fit to the data at each contraction. C, MU discharge rate 611 as a function of the threshold force at each contraction with regression lines superimposed on 612 motor units in a representative subject. Each data point represents 1 MU. Group data $(n=13)$ 613 showing the fitted slopes are also shown.

614 Figure 5. A, First common component (FCC) and force. Comparison between the FCC and the 615 force signal during unilateral and bilateral contractions in a representative subject. B, graph 616 showing correlation between the CVs for the force and FCC signal. Note that individuals with 617 more force fluctuation were the ones with larger variability in low-frequency oscillations of MU 618 discharge rate.

619 Figure 6. Graph showing correlation between the CVs for the force and FCC signal. Note that 620 individuals with more force fluctuation were the ones with larger variability in low-frequency 621 oscillations of MU discharge rate. 

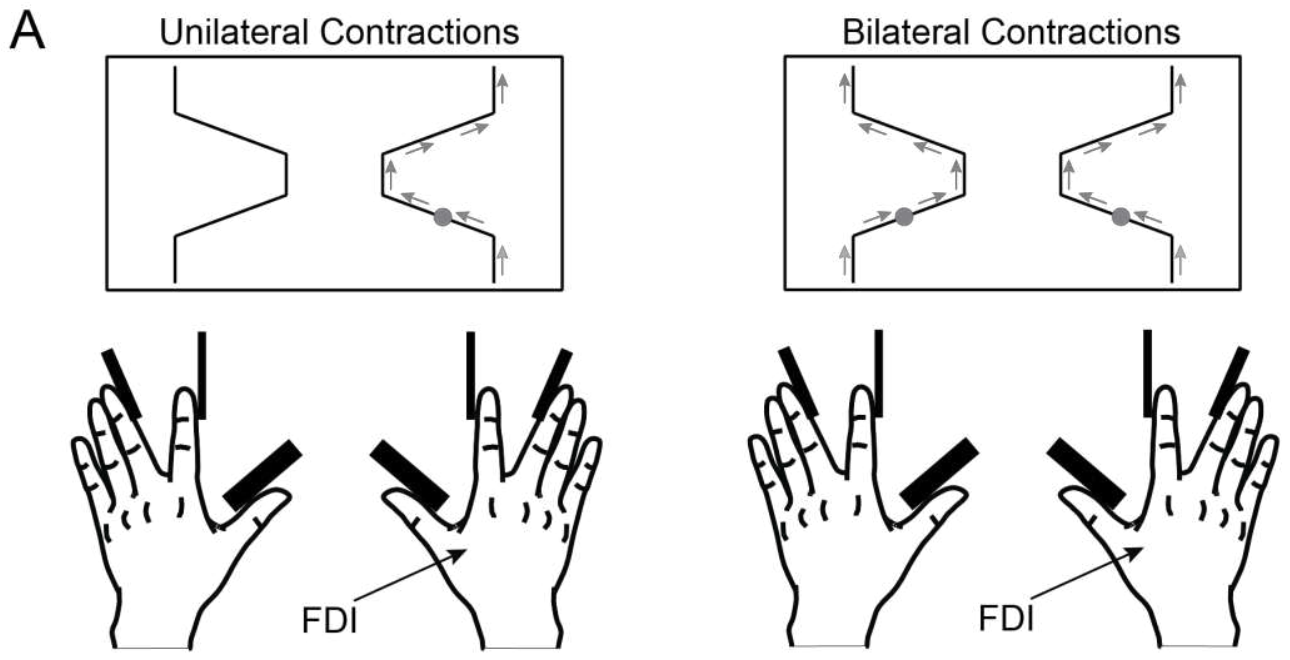

B

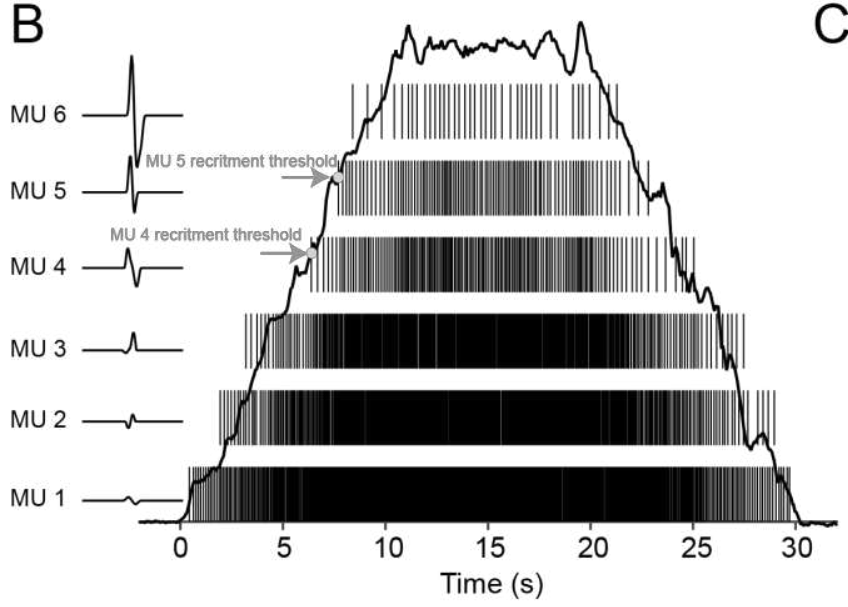

$C_{20} \mathrm{MU} 6$

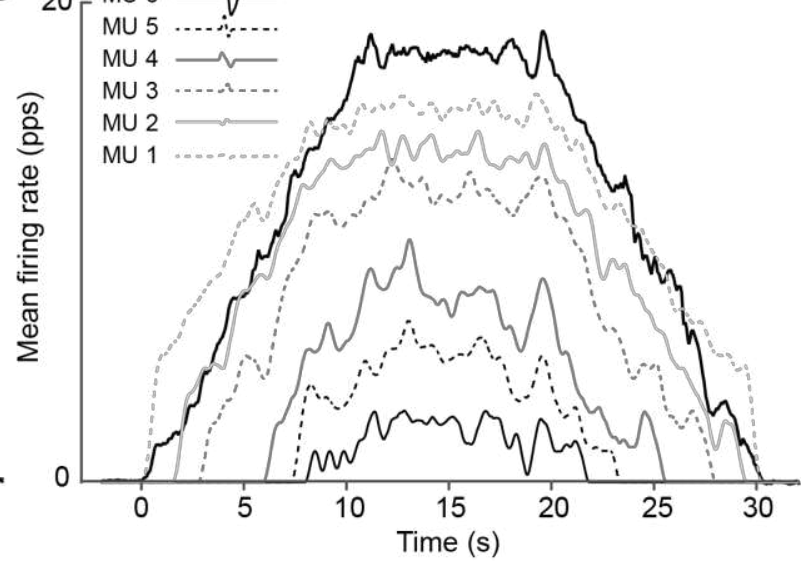



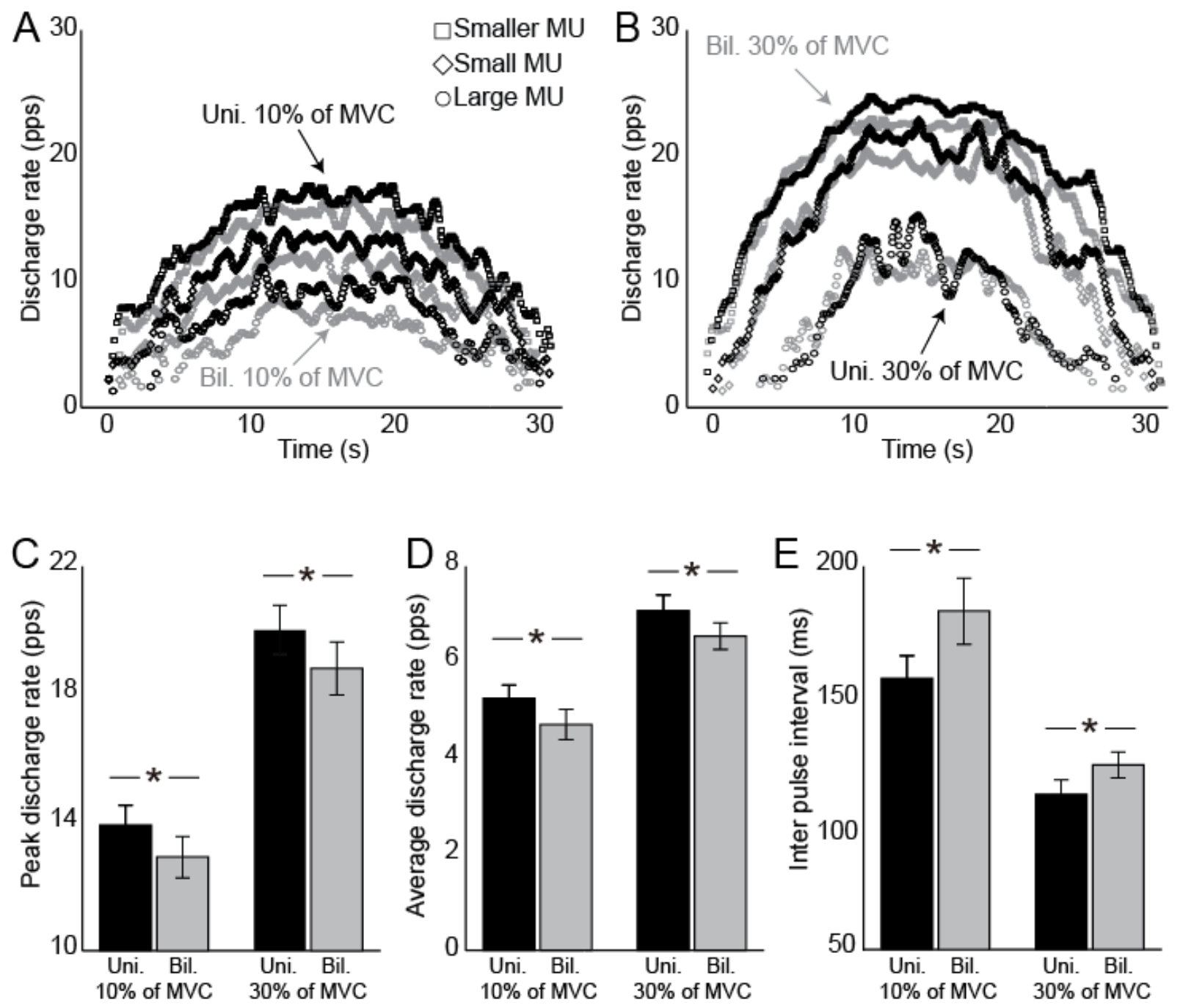


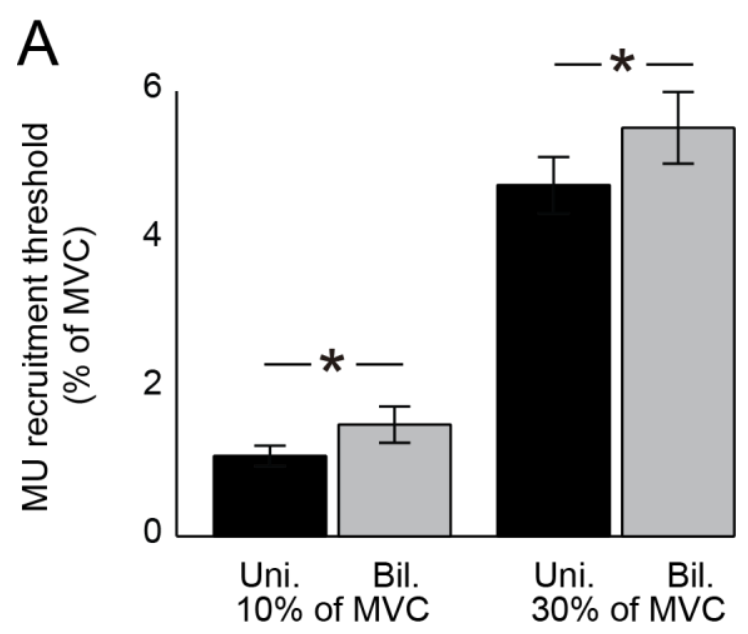

B

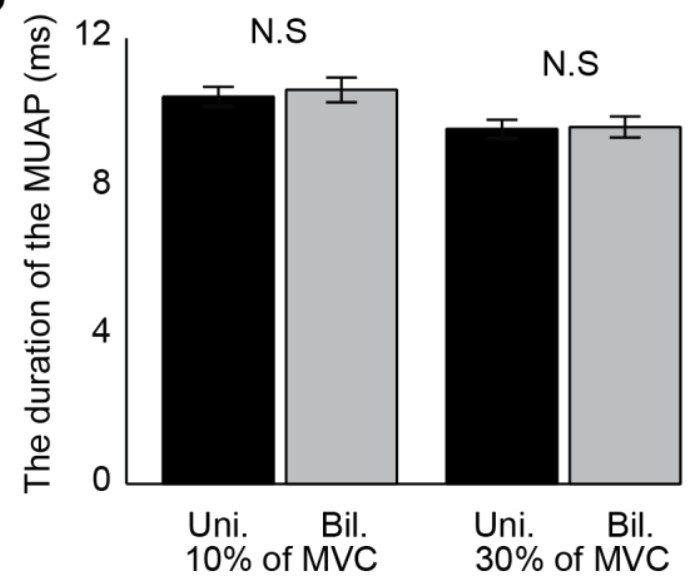

C

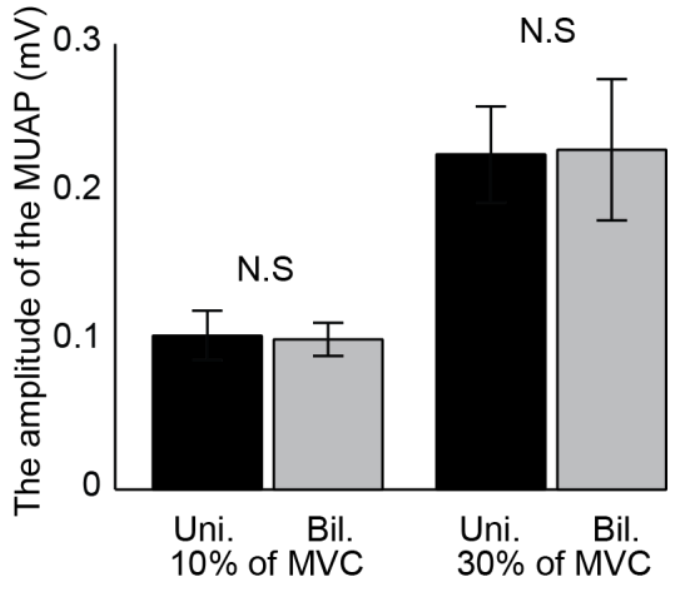



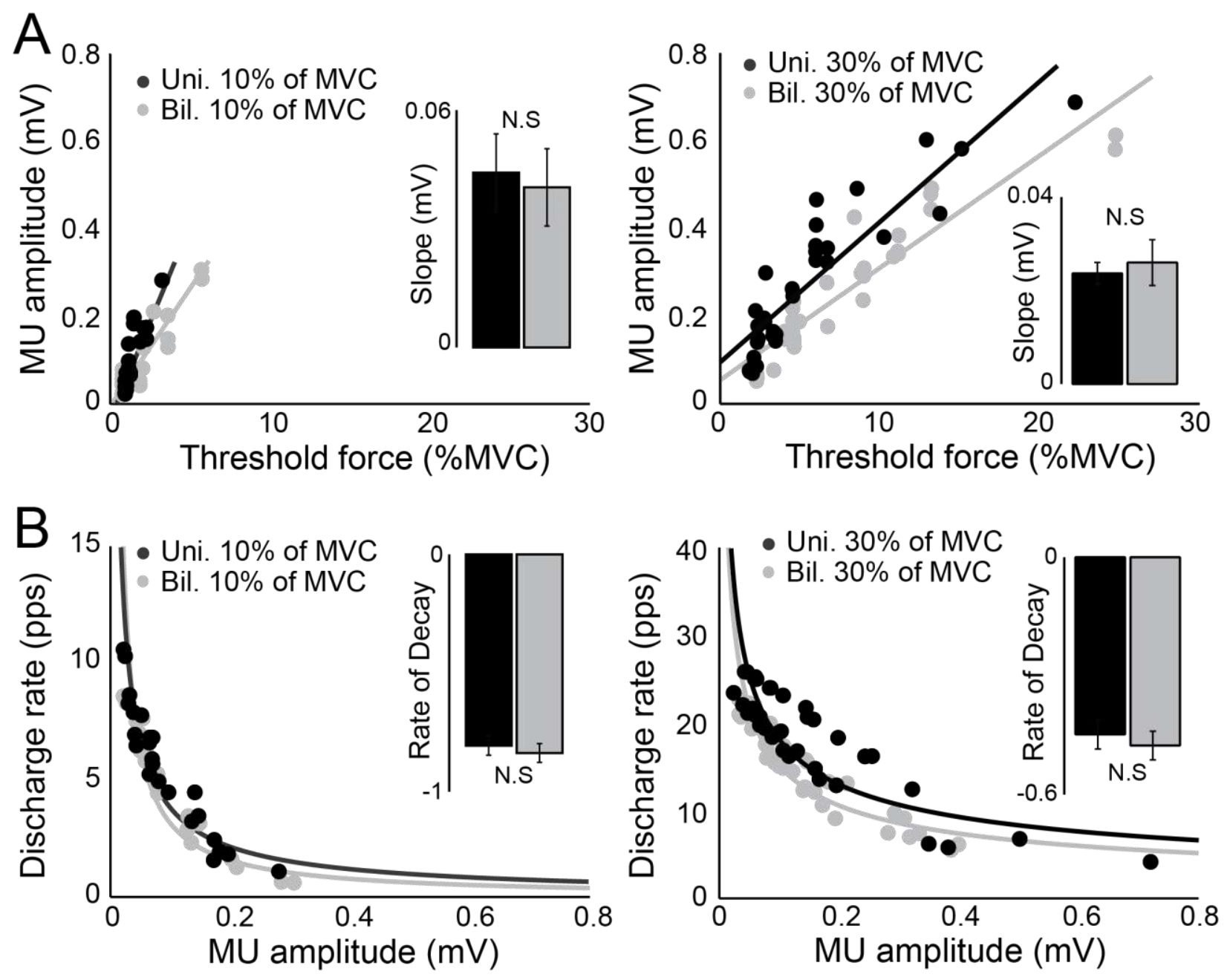

639
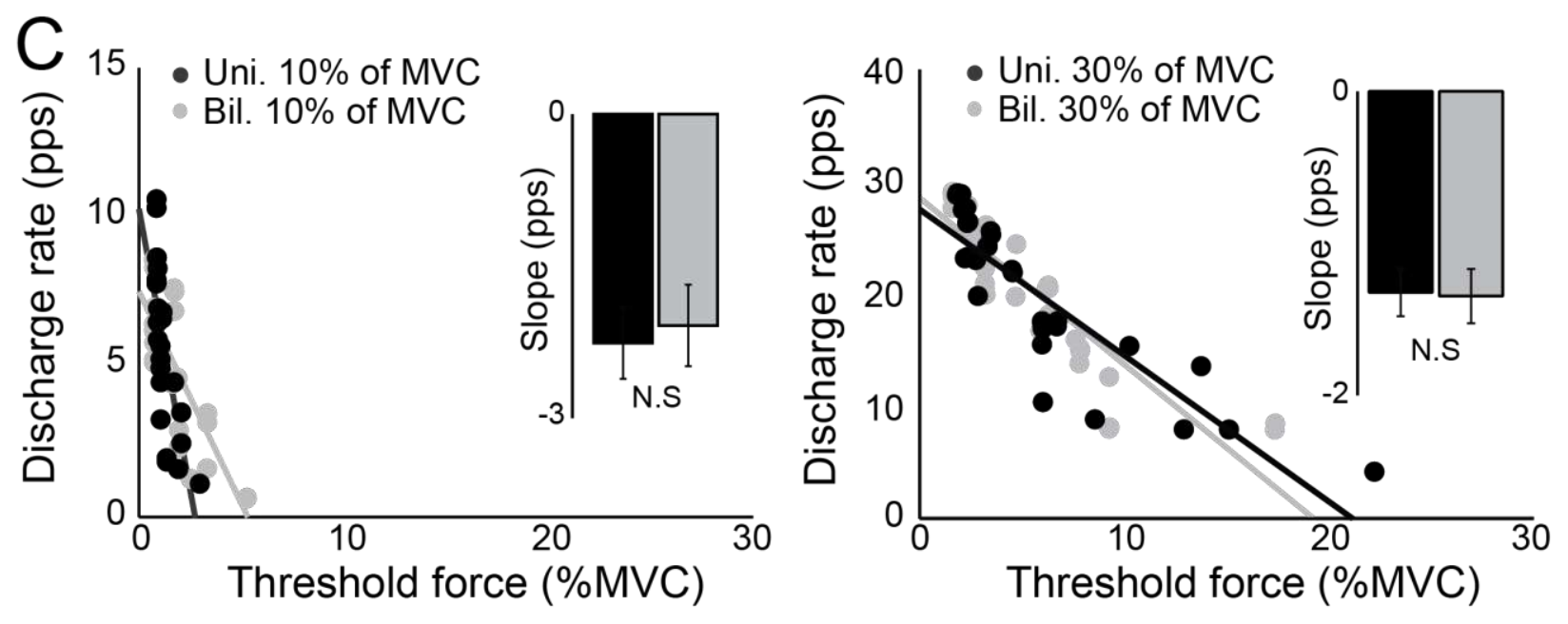
641

A

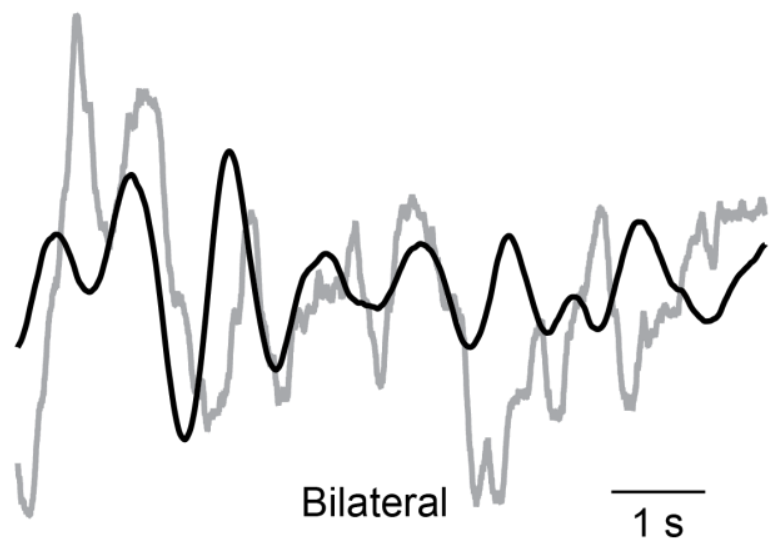

642

643

644

645

646
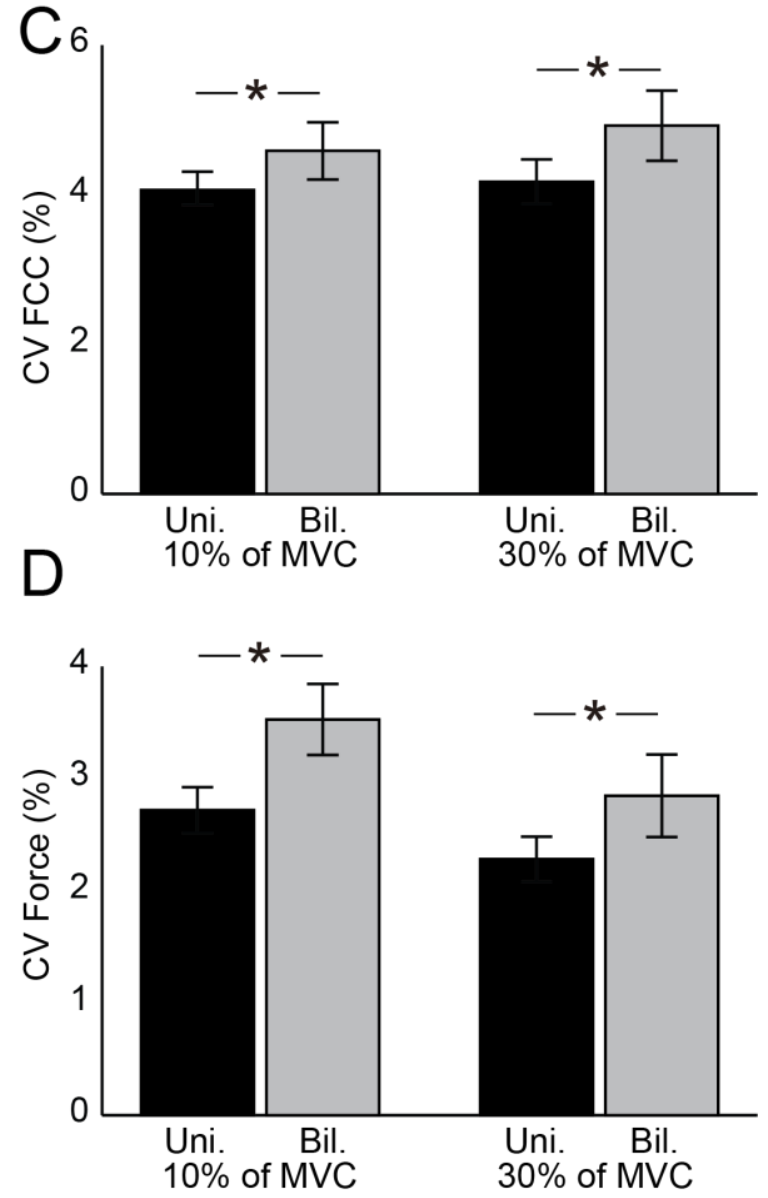
Figure 6

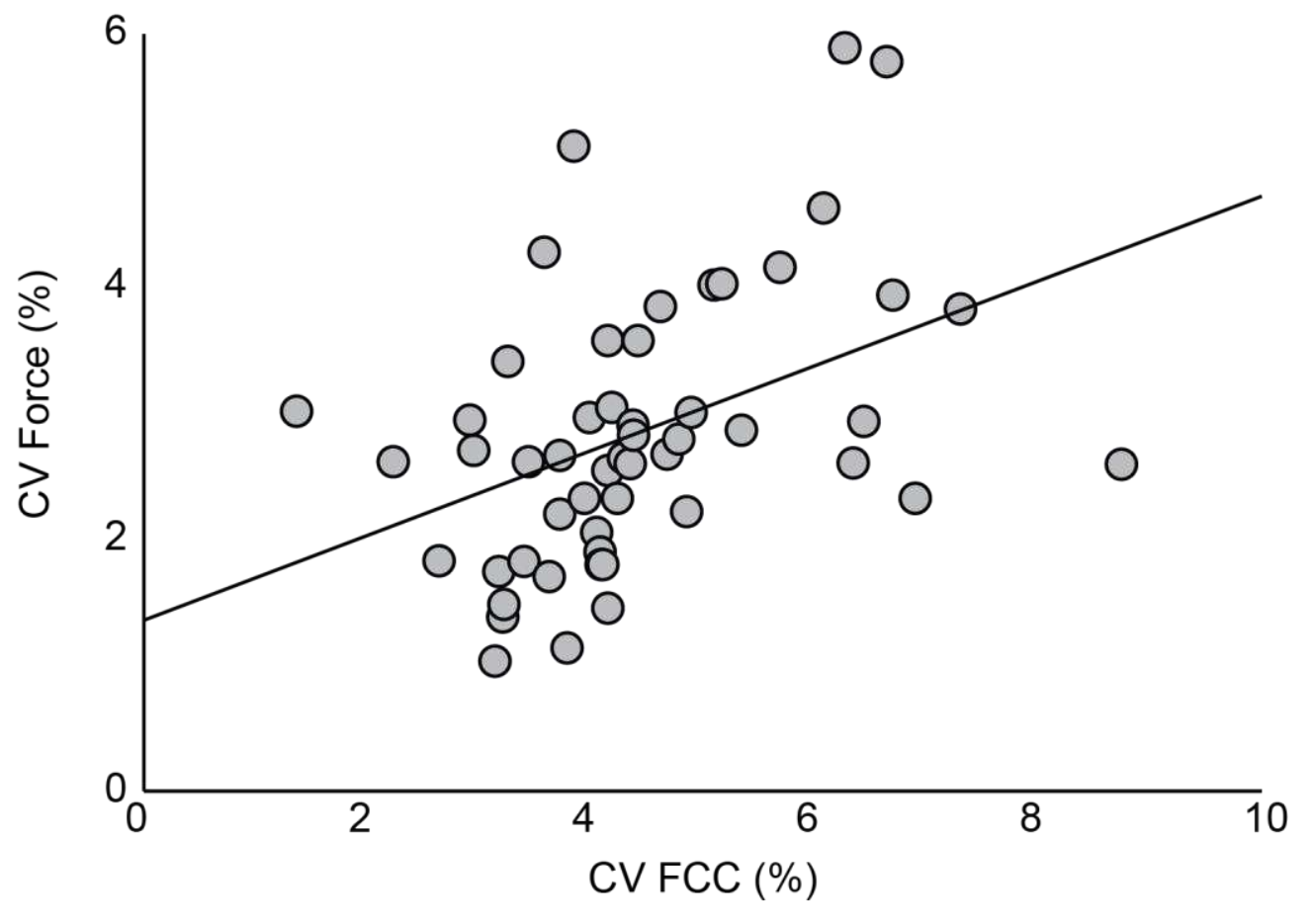

